\section{Sutherland, Earl Wilbur}

\section{W. Hubl}

Ehem. Krankenhaus Dresden-Friedrichstadt, Institut für Klinische Chemie und Labormedizin, Dresden, Deutschland

Lebensdaten Amerikanischer Mediziner, geboren am 29. November 1915 in Burlingame (Kansas, USA), gestorben am 9. März 1974 in Miami (Florida). Er studierte in Topeka, der Hauptstadt seines Heimatstaates, von 1933 bis 1937 Medizin, promovierte im Jahr 1942 an der Washington University in Saint Louis und wurde 1953 als Professor für Pharmakologie an die Western Reserve University in Cleveland (Ohio) berufen. Von 1963 bis 1973 lehrte Sutherland als Professor für Physiologie an der Vanderbilt University in Nashville (Tennessee) und ging dann an die Universität in Miami.

Verdienste Sutherland entdeckte im Jahr 1957 das zyklische Adenosinmonophosphat. Er erlangte wissenschaftliche Aner- kennung mit seinen Arbeiten über hormongesteuerte Zellaktivitäten. Er entwickelte das Second-Messenger-System, wobei die Wirkung der Hormone von der Zellmembran an den ,second messenger“, das zyklische Adenosinmonophosphat, übertragen wird. Sutherland erhielt im Jahr 1971 den Nobelpreis für Physiologie und Medizin für seine Entdeckungen über die Wirkungsmechanismen von Hormonen. Earl Sutherland erhielt zahlreiche weitere Ehrungen einschließlich des Albert-Lasker-Preises (1970) und der National Medal of Science (1973).

\section{Literatur}

Herder WW (2014) Heroes in endocrinology: nobel prizes. Endocr Connect 3:R99

Lindsten J (1992) Nobel lectures, physiology or medicine 1971-1980. World Scientific Publishing Co., Singapore

Raju TN (1999) The Nobel chronicles. 1971: Earl Wilbur Sutherland, Jr. (1915-74). Lancet 354(9182):961 